\title{
Identification of novel biomarkers in ischemic stroke: a genome-wide integrated analysis
}

Qizhi Xie ${ }^{1,2}$, Xiaoyun Zhang ${ }^{1,2}$, Sijia Peng ${ }^{1}$, Jingjing Sun ${ }^{1}$, Xiao Chen ${ }^{1,2}$, Yuanfei Deng ${ }^{1,3}$ and Li Yi ${ }^{1 *}$

\begin{abstract}
Background: Ischemic Stroke (IS) is the most common neurological emergency disease and has become the second most frequent cause of death after coronary artery disease in 2015. Owing to its high fatality rate and narrow therapeutic time window, early identification and prevention of potential stroke is becoming increasingly important.
\end{abstract}

Methods: We used meta-analysis and bioinformatics mining to explore disease-related pathways and regulatory networks after combining messengerRNA (mRNA) and miRNA expression analyses. The purpose of our study was to screen for candidate target genes and microRNA(miRNA) for early diagnosis of potential stroke.

Results: Five datasets were collected from the Gene Expression Omnibus (GEO) database by systematical retrieval, which contained three mRNA datasets (102 peripheral blood samples in total) and two miRNA dataset (59 peripheral blood samples). Approximately 221 different expression(DE) mRNAs (155 upregulated and 66 downregulated mRNAs) and 185 DE miRNAs were obtained using the metaDE package and GEO2R tools. Further functional enrichments of DE-mRNA, DE-miRNA and protein-protein interaction (PPI) were performed and visualized using Cytoscape.

Conclusion: Our study identified six core mRNAs and two regulated miRNAs in the pathogenesis of stroke, and we elaborated the intrinsic role of systemic lupus erythematosus (SLE) and atypical infections in stroke, which may aid in the development of precision medicine for treating ischemic stroke. However, the role of these novel biomarkers and the underlying molecular mechanisms in IS require further fundamental experiments and further clinical evidence.

Keywords: Ischemic stroke, Bioinformatics, Biomarkers, Genes, miRNA, Atypical infection

\section{Background}

Stroke is the most common neurological emergency disease and has become the second leading cause of death after coronary artery disease in 2015 , leading to 6.3 million deaths [1]. In addition, stroke is also a leading cause of long-term disability. The pathophysiological hallmarks

\footnotetext{
*Correspondence: yilitj@hotmail.com

'Department of Neurology, Peking University Shenzhen Hospital, Shenzhen, China

Full list of author information is available at the end of the article
}

of ischemic stroke involve part of the brain losing blood supply, which initiates the ischemic cascade. Brain tissue ceases to function if oxygen deprivation persists for 60 to $90 \mathrm{~s}$, and will suffer irreversible death of brain cells occurs after approximately $3 \mathrm{~h}$. The primary risk factor for stroke is hypertension; other risk factors include smoking, obesity, hyperlipidemia, diabetes, previous transient ischaemic attack and atrial fibrillation [2] Stroke is characterized by neurological defect signs and symptoms, including hemiplegia, hemianesthesia, 
difficulty in speaking and understanding or loss of vision on one side. Even after intensive therapy, certain symptoms can be permanent, affecting $75 \%$ of stroke survivors and rendering them unable to manage their daily lives [3].

Stroke was originally deemed to be a sporadic disease. However, several epidemiology studies have shown that the morbidity of stroke or transient ischaemic attack was $12.3 \%$ among first degree relatives of stroke patients (vs $7.5 \%$ in the control group) [4], and the prevalence of stroke in offspring was shown to be three times higher if a parent had a stroke before 65 years of age [5]. Currently, it is widely believed that stroke is a complex multifactorial disease that is caused by interactions among blood vessels and environmental and genetic factors. Pathogenic mutations, such as Neurogenic locus notch homolog protein 3(NOTCH3) gene and HtrA Serine Peptidase 1(HTRA1) gene, have been reported in certain types of monogenetic stroke syndromes, such as cerebral autosomal dominant arteriopathy with subcortical infarcts and leukoencephalopathy(CADASIL) and cerebral autosomal recessive arteriopathy with subcortical infarcts and leukoencephalopathy(CARASIL). In addition, certain molecular genetic variations have been shown to be closely related to ischemic stroke, such as Paired-like homeodomain transcription factor 2 (PITX2), Histone deacetylase 9 (HDAC9), and Zinc finger homeobox protein 3 (ZFHX3) [5]. However, most of these gene mutations may exist as susceptibility genes, cooperating with other risk factors to cause the disease.

In addition to a single gene mutation, epigenetic mechanisms, such as DNA methylation, histone modifications and regulation by miRNAs, can also influence gene expression, which makes it difficult to analyze this disease, particularly sporadic stroke. Moreover, miRNA has been reported as a vital regulatory mechanism for the recovery of stroke [6] and has also been associated with the death of neurons and the repair of damaged tissue in the case of cerebral infarction [7]. Since the relationship between a given mi-RNA and its target genes is one-to-many rather than one-to-one, the mutual regulatory network between them may offer us a unique perspective to understand the disease and may provide potential therapeutic targets.

The massively parallel microarray technique can be applied to identify variant gene expression and pathways. This technique is used to investigate the relationship between gene expression and phenotypic differences and to gain deeper insights into the pathogenesis of complex diseases [8]. Bioinformation mining allows for the categorization and detection of large-scale genetic data according to phenotypic characteristics, potentially leading to novel hypotheses about the underlying mechanisms [9]. However, genome-wide expression data have limitations, such as small sample sizes, including poor repeatability and contradictory results. To take advantage of the big data era and reduce the limitations due to a small sample size, data from multiple datasets and platforms were collected in our integrated analysis.

The purpose of our study was to screen for candidate target genes and miRNAs in stroke using meta-analysis and bioinformatic mining and to explore disease-related pathways and regulatory networks after combining the mRNA and miRNA expression analyses.

Our study identified six core mRNAs and two regulated miRNAs in the pathogenesis of stroke, and we elaborated the intrinsic role of SLE and atypical infections in stroke.

\section{Methods}

\section{Data collection and pre-processing}

Expression profile data associated with stroke were obtained from Gene Expression Omnibus (GEO), which is a public functional genomic data repository. Ischemic stroke-related datasets were retrieved using the keyword "stroke" of Homo sapiens (organisms). Using the cutoff date August 15, 2018, 1037 datasets were retrieved. The inclusion criteria were as follows: (1) original experimental studies; (2) peripheral blood sample data provided; (3) mRNA expression profile provided; (4) access to the raw data (CEL files); and (5) the required diagnostic criteria for ischemic stroke are fulfilled. The exclusion criteria were as follows: (1) non-ischemic stroke sample; (2) repeated uploading of datasets; and (3) retrospective analysis. All of the included analyses were verified by the ethics committee. Pre-processing programs (including background adjustment, normalization, summarization, gene chip probe annotation) were executed using $\mathrm{R}$ language. CEL files were loaded using library (affy) to read the signal diagrams. We use the RMA algorithm on Bioconductor software to process all raw data files to obtain the expression value of each gene chip. For the miRNA microarray, qualified human plasma miRNA datasets were imported into the online tool GEO2R.

\section{Quality control and DE-mRNA screening}

For quality control (QC), we used the Relative Log Expression (RLE) method to load the included mRNA expression datasets. RLE establishes a reference array that is generated from the median of all arrays for each probe set, and the expression value of each sample was normalized. Most of the expression values are supposed to be stable with respect to the median and should be approximately 0 , accordingly.

The "Batch effect" is a type of non-biological expression variation that is found across multiple batches of microarray analysis, making it difficult to combine data for an integrated analysis. 
Johnson WE et al. proposed parametric and nonparametric empirical Bayes frameworks to adjust data for batch effects that are robust to outliers in small sample sizes, making them comparable to large sample methods [10]. We used this method to remove the batch effects using the Surrogate Variable Analysis (SVA) package in $\mathrm{R}$ studio to make the data more suitable for comparisons.

The Linear Model for Microarray (LIMMA) package was used to pool the eligible microarray data to acquire DE-genes in stroke. In LIMMA, $P$-values were extrapolated with a modified two sample t-test, and Fisher's method was implemented to analyze differences between two groups [11]. A corrected P-value $(P<0.05)$ and $\log$ Fold Change $>1.4$ were considered to be statistically significant for DE mRNAs in stroke, and a false discovery rate (FDR) of 0.05 was used to correct for multiple testing. For visualization, DE mRNAs were plotted using the MetaDE package.

\section{Enrichment analysis of stroke-related DE genes}

Online tools, such as Database for Annotation, Visualization and Integrated Discovery (DAVID), Kyoto Encyclopedia of Genes and Genomes (KEGG) pathways, Gene Ontology (GO) terms and Genetic Association Database (GAD) were used to predict the prospective function and further functional categories [12-14]. $P<0.05$ was considered significant in the enrichment analysis. Given that proteins are the biomacromolecules that execute functions in our bodies, the STRING database [15] was applied for the critical assessment by visualizing the Protein-Protein Interactions (PPI) using Cytoscape [16].

\section{Gene set enrichment analysis}

Gene Set Enrichment Analysis (GSEA) is an advanced method for determining whether an a priori defined set of genes shows statistically significant, consistent differences between two biological groups [17]. This method has advantage due to focusing on gene sets, that is, groups of genes that share a common biological function, chromosomal location, or regulation. This method can avoid the limitations of the common enrichment approach, which focuses on a handful of genes at the top of $\mathrm{L}$, that is, those genes which exhibit the largest difference.

\section{Analysis of the miRNA expression dataset and target prediction}

GEO2R (http://www.ncbi.nlm.nih.gov/geo/geo2r/) is an easy-to-use online tool for identifying differential expression in miRNA series. GEO2R automatically calculates the false discovery rate (FDR) and detects statistically significant genes $(p<0.05)$ simultaneously with FDR correction by using multiple t-test.

The target genes of miRNAs were predicted using miRNet, which is a comprehensive tool suite that enables the statistical analysis and functional interpretation of data generated from current miRNA studies.

A simplified flowchart (Fig. 1) illustrates the abovedescribed process.

\section{Results \\ Coanfluence analysis of ischemic stroke gene expression datasets}

Three primary datasets with available mRNA expression data for PBL samples in stroke patients were identified by searching the GEO database (GSE66724, GSE58294, GSE22255). The detail of the participants are provided in additional file 2 . After quality control using RLE and the removal of the batch effect (Fig. 2), a total of 102 PBL samples (51 patients and 51 controls, Table 1) were pooled into the DE-gene analysis. Approximately 221 DE mRNAs were identified (155 upregulated mRNAs and 66 downregulated mRNAs). A heatmap of the top 20 DE mRNAs was generated by setting a specific FDR and fold change value (Fig. 3). The details of each DE mRNA are given in Supplementary table (see additional file 1).

\section{Enrichment analysis of the DE-mRNAs}

Through the enrichment analysis of Genetic Associated Disease, type 2 diabetes, chronic renal failure, Alzheimer's disease, coronary artery disease, atherosclerosis, myocardial infarction, lung cancer, asthma, high-density lipoproteincholesterol(HDL-C) level, asthma and obesity were deemed important in stroke (Fig. 4a). In the KEGG pathway enrichment analysis, DE-mRNAs were primarily involved in viral carcinogenesis, alcoholism, the tumor necrosis factor(TNF)signal pathway, the Nuclear Factor-KappaB(NF-kap$\mathrm{paB})$ pathway and the SLE pathway. The enrichment results using the Gene Ontology(GO) database in three categories were as follows: (1) biological processes: plasma membrane, extracellular exosome and neuron projection (Fig. 4b); (2) cellular component: inflammatory response, negative regulation of cell proliferation and positive regulation of angiogenesis (Fig. 4c); (3) molecular functions: calcium ion binding, carbohydrate binding and protease binding (Fig. 4d). The above-mentioned enrichment analysis revealed that DE-genes were primarily related to common risk factors, such as type 2 diabetes, coronary artery disease and atherosclerosis, and the general event was the activation of the TNF-signaling pathway (Fig. 4e). Furthermore, we identified a pathway (the SLE pathway) that has seldom been reported to be closely 


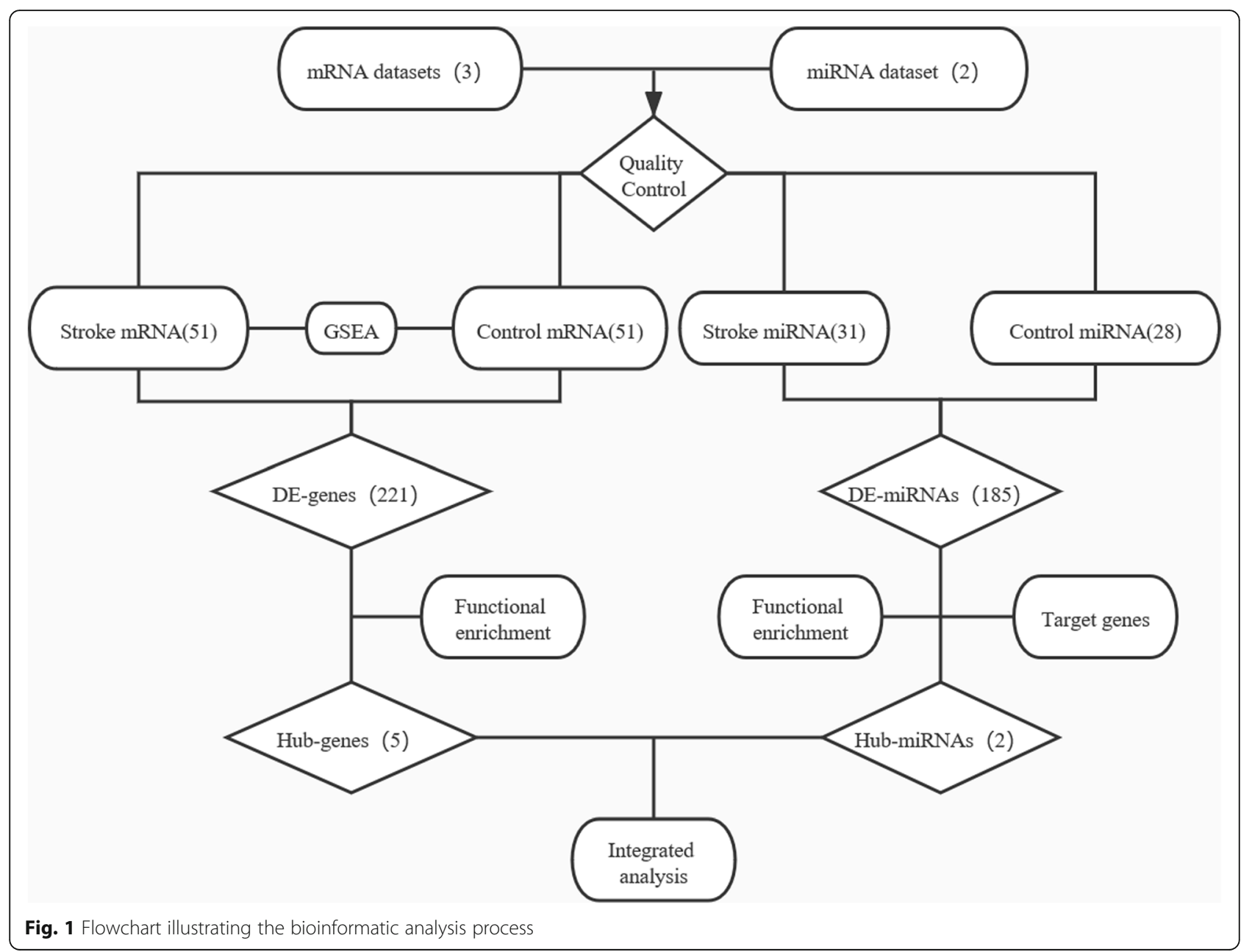

associated with stroke, which was consistent with the results of the GO enrichment with immune response biological process.

In the GSEA, we identified six pathways that were enriched (Fig. 5). These pathways included epithelial cell signaling in HP infection, vibrio cholerae infection, histidine metabolism, complement and coagulation cascades, systemic lupus erythematosus and the toll-like receptor signaling pathway. Notably, the SLE pathway (Fig. 6) was identified in both the DEgene analysis and the GSEA analysis, indicating that this pathway may be strongly related to stroke.

To further investigate the functions and interactions of the upregulated DE-mRNAs (Fig. 7) and all DE-mRNAs, we used the STRING database to construct two PPI networks, and the results were imported into Cytoscape and visualized. Interestingly, six genes (PTGS2, IL1B, STAT3, MMP9, SOCS3 and CXCL1) were located in the central position of the PPI networks. The significance level is shown in Table 2. Of these core genes, five of them (excluding STAT3) were linked to the TNF signaling pathway.

\section{Analysis of the stroke miRNA expression dataset}

Dataset GSE86291 and GSE55937 were available miRNA expression datasets containing 59 plasma samples. 31 samples were from stroke patients, and 28 were from control groups. After QC (Fig. S1), a total of 185 DEmiRNAs were identified using the online tool GEO2R(74 DE-miRNAs from GSE86291 and 111 DE-miRNAs from GSE55937). Since they were generated from different platform, we were not supposed to compare the data directly. But they actually shared one miRNA in common, that is has-miR-3135b. Among GSE86291, the top six most significant DE-miRNAs were hsa-miR-140-3p, hsa-miR-320b, hsa-miR-320d, hsa-miR-320e, hsa-miR5100 and hsa-miR-30d-5p (Table 3). An experimentally supported miRNA database (miRBase) was used to predict the target genes of the identified miRNAs base on experimental verification and various prediction algorithms. Target genes that were regulated by two or more DE-miRNAs were included to form the miRNA-target gene-pathway network (Fig. 8 and Fig. 9). Through this network, three pathways, including the Neurotrophin signaling pathway, Mitogen-activated protein 


\section{RLE}

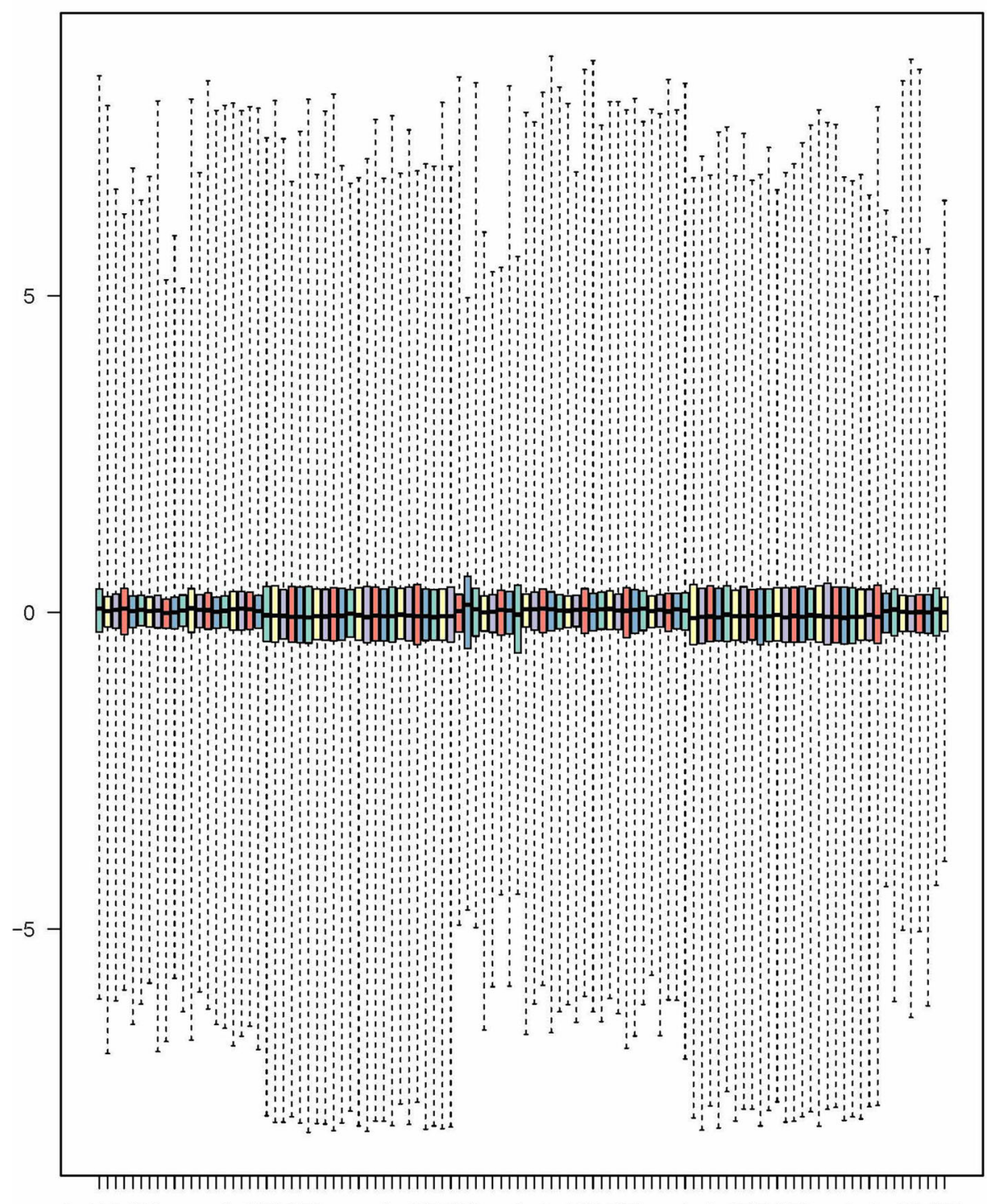

controlA1.CEL controlA9.CEL controlB5.CEL strokeA15.CEL strokeB15.CEL strokeC3.CEL

Fig. 2 Relative Log Expression (RLE) signal graph

Table 1 Baseline characteristics of datasets

\begin{tabular}{|c|c|c|c|c|c|c|c|c|}
\hline \multirow[t]{2}{*}{ Study } & \multirow[t]{2}{*}{ Country } & \multirow{2}{*}{$\begin{array}{l}\text { GEO } \\
\text { accession }\end{array}$} & \multirow[t]{2}{*}{ Platform } & \multirow{2}{*}{$\begin{array}{l}\text { Sample } \\
\text { type }\end{array}$} & \multirow{2}{*}{$\begin{array}{l}\text { Experiment } \\
\text { type }\end{array}$} & \multicolumn{3}{|c|}{ Case/Control } \\
\hline & & & & & & Number & Age $($ mean $\pm S D)$ & Male\% \\
\hline Allende M & Spain & GSE66724 & GPL570 & $\mathrm{PBL}^{\mathrm{a}}$ & mRNA & $8 / 8$ & / & / \\
\hline Stamova B & USA & GSE58294 & GPL570 & PBL & mRNA & $23 / 23$ & $71.7 \pm 7.9 / 57.9 \pm 3.3$ & $52.2 / 52.2$ \\
\hline Krug $T$ & Portugal & GSE22255 & GPL570 & PBL & mRNA & $20 / 20$ & $60.2 \pm 10.6 / 58.7 \pm 11.0$ & $50.0 / 50.0$ \\
\hline Tian C & China & GSE86291 & GPL18402 & Plasma & microRNA & $7 / 4$ & $64.4 \pm 16.5 / 59.8 \pm 4.0$ & $85.7 / 75.0$ \\
\hline Jickling GC & USA & GSE55937 & GPL16384 & PBL & microRNA & $24 / 24$ & $62.4 \pm 8.1 / 63.8 \pm 8.5$ & $50 / 50$ \\
\hline
\end{tabular}




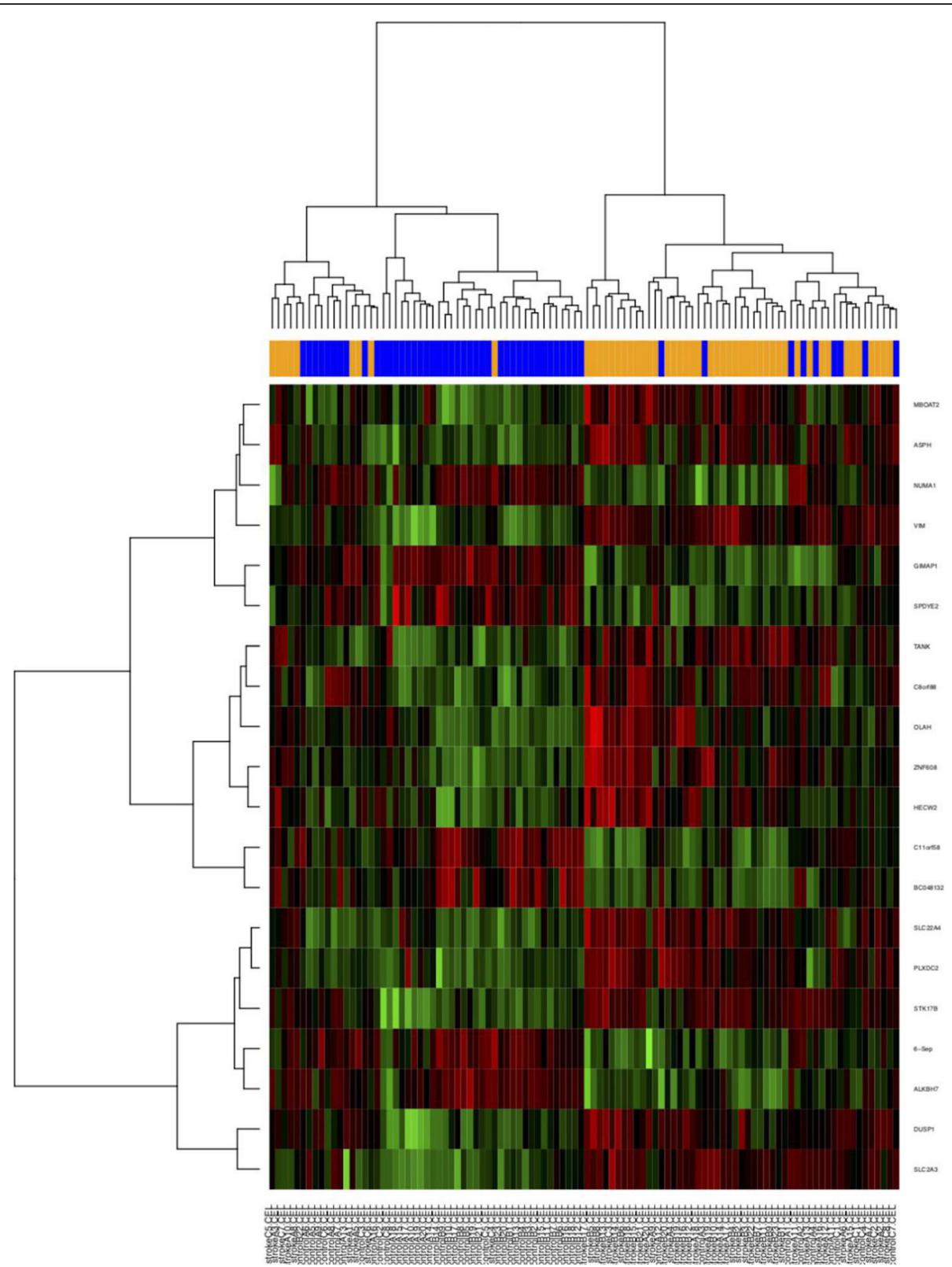

Fig. 3 Heatmap of the top 20 differentially expressed genes (for the sake of space, only a portion of the figure is shown here)

kinase(MAPK) signaling pathway and Shigenllosis infection were presented. Notably, miR-320b and miR-320d had the most common target genes, which made these miRNAs the center of the regulatory network.

\section{Comprehensive analysis of DE genes and miRNAs}

Overall, we identified potentially useful biomarkers, six mRNAs and two miRNAs, as well as several novel pathways (the SLE pathway and atypical infection pathways) as a matter of priority.

\section{Discussion}

Cross-country studies of ischemic stroke gene expression datasets were standardized and integrated in our study using a precise method for further integrated analysis. The purpose of our work was to reduce the bias of sample studies and to screen for significant susceptibility genes that may be used to predict the potential for stroke. We used the MetaDE package in $\mathrm{R}$ language to merge and filter gene expressions [18]. A total of 155 upregulated DE mRNAs in PBL samples of stroke and $185 \mathrm{DE}$ miRNAs were identified. Of these, we identified 


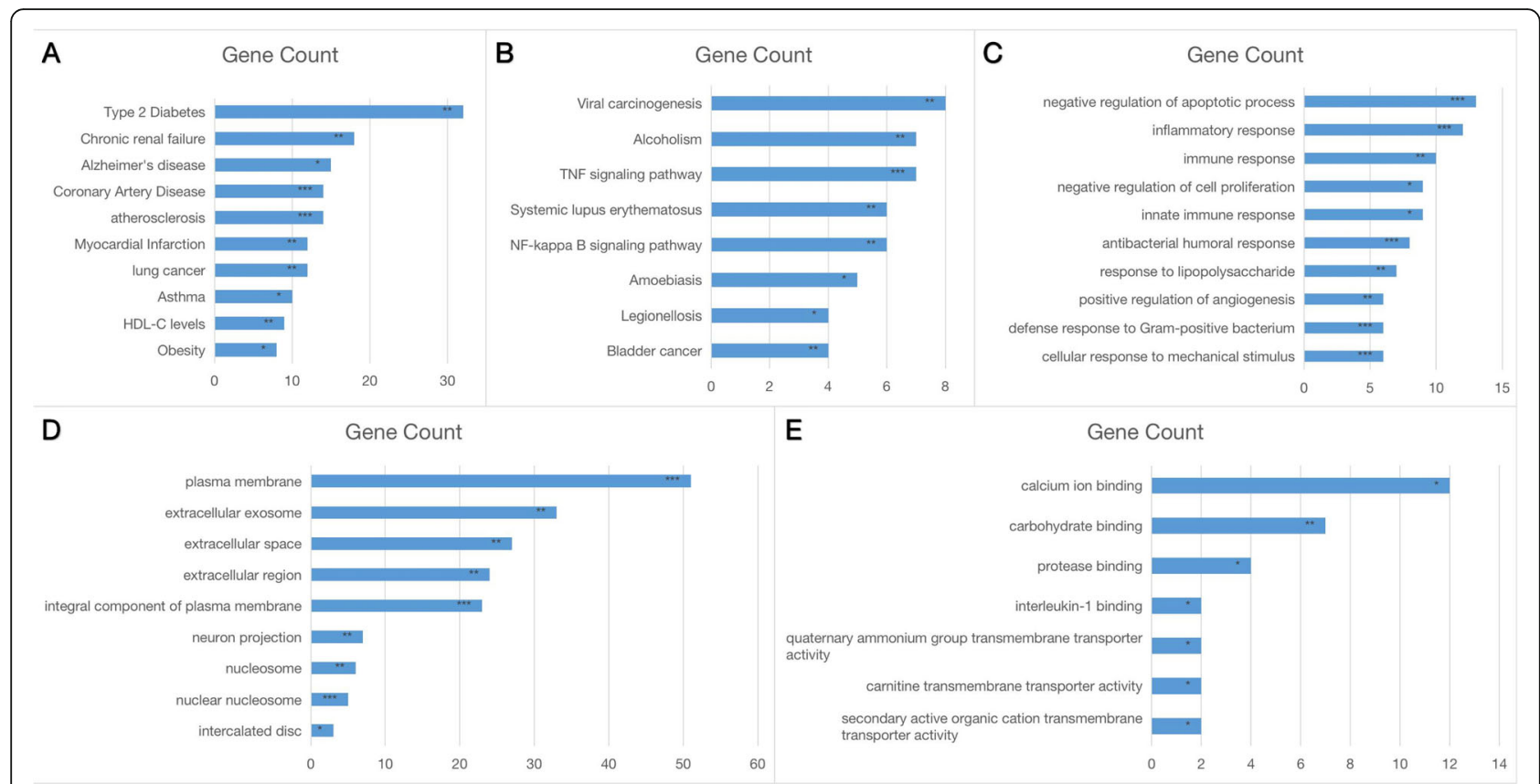

Fig. 4 Functional enrichment analysis of meta-DE genes. (A) GAD-disease analysis. (B) KEGG pathway enrichment analysis. (C) Cellular components of $\mathrm{GO}$ enrichment analysis. (D) Biological processes of $\mathrm{GO}$ enrichment analysis. (E) Molecular functions of $\mathrm{GO}$ enrichment analysis

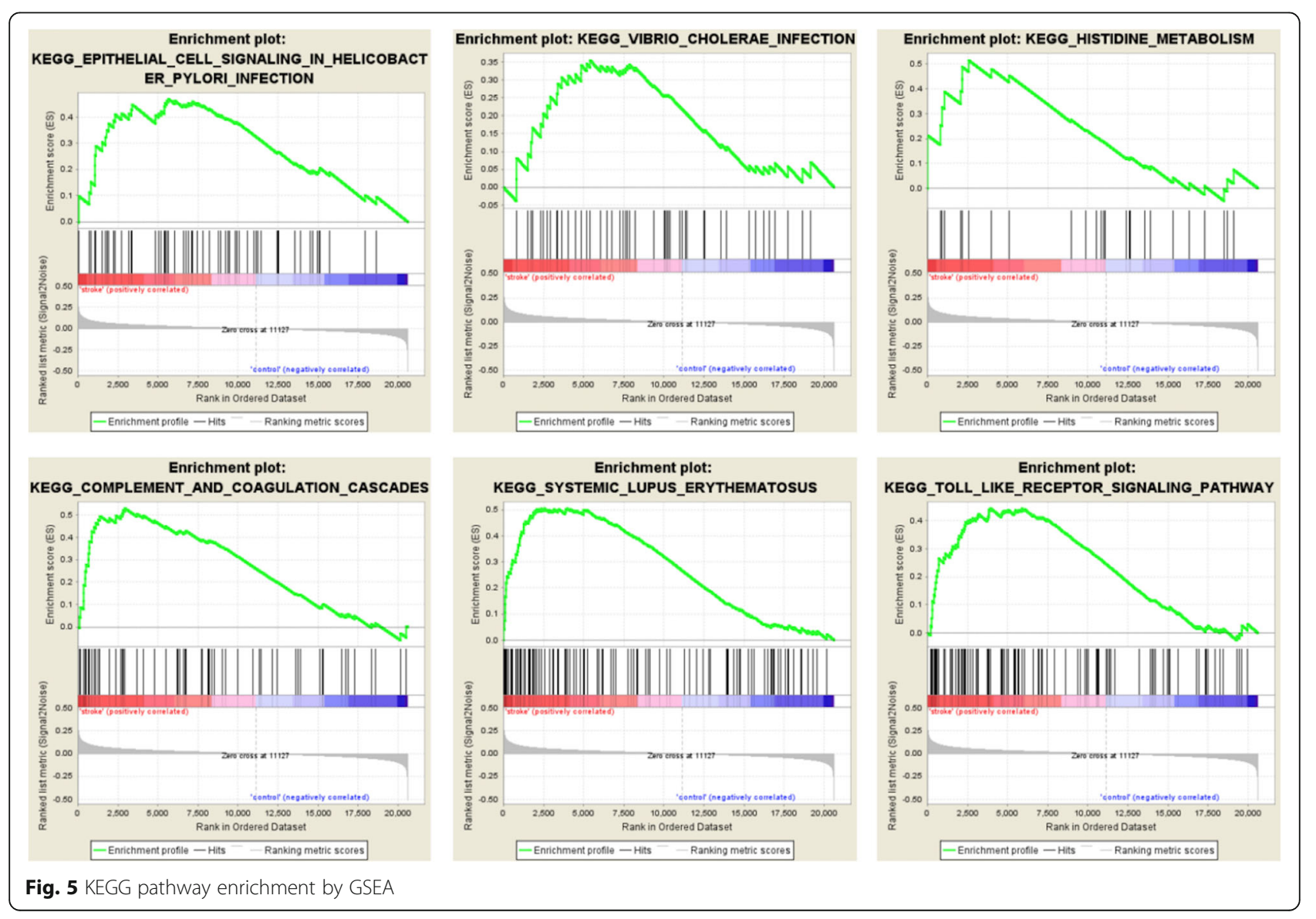




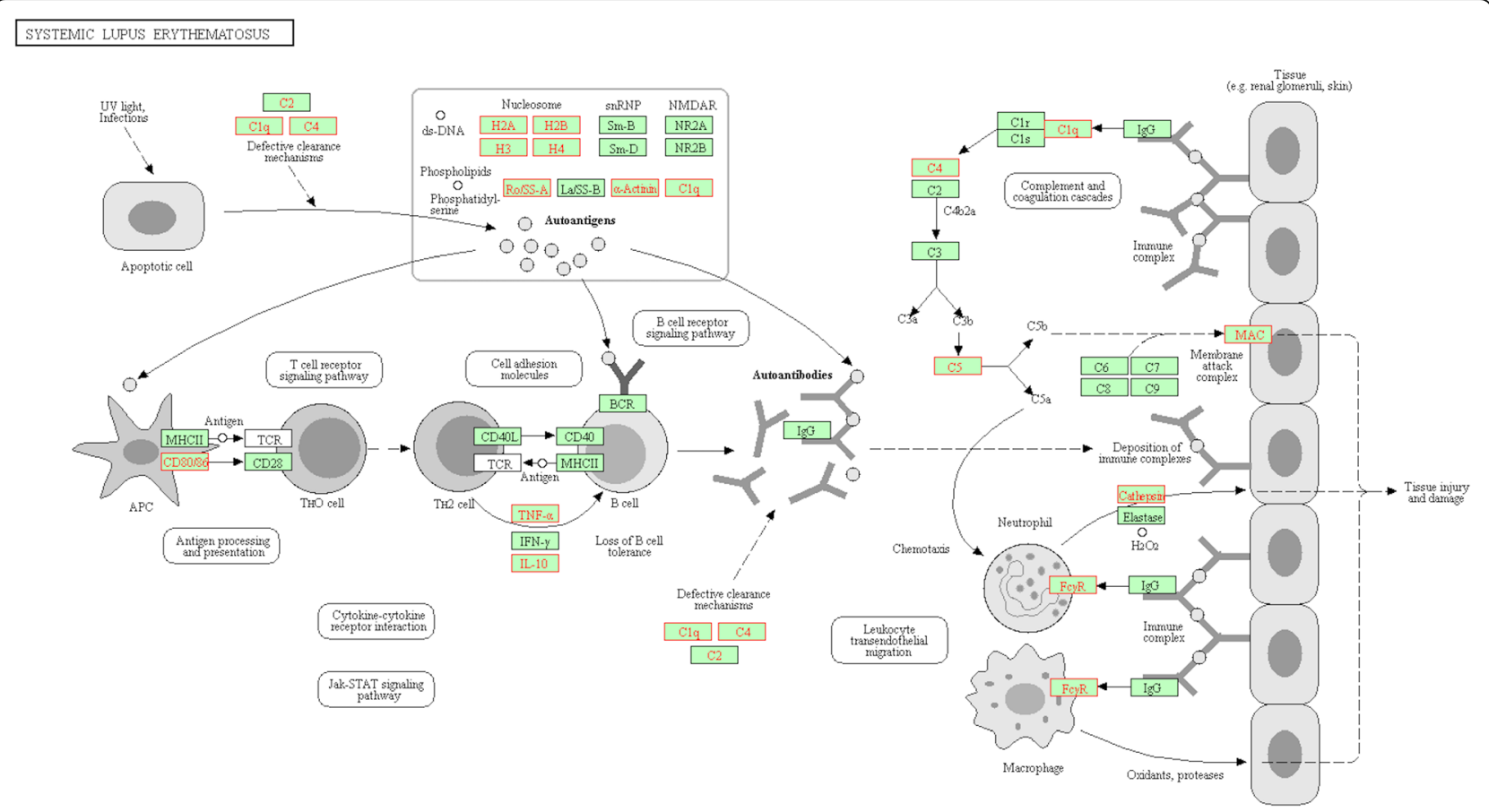

Fig. 6 Systemic lupus erythematosus pathway in KEGG. The core enrichment genes identified in GSEA are shown in red
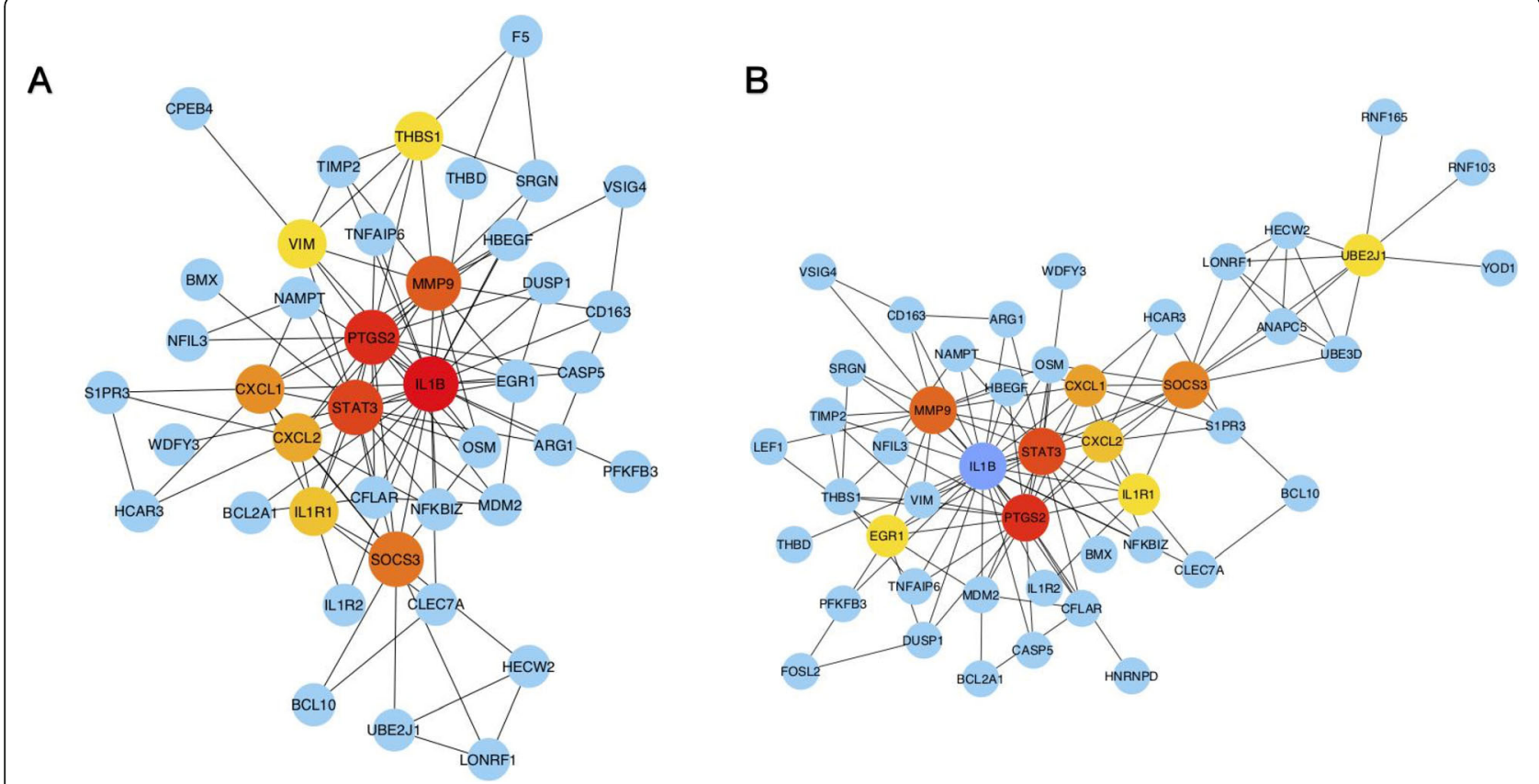

Fig. 7 PPI networks. (A) PPI network of upregulated DE-mRNAs. (B) PPI network of all DE-mRNAs. The size and color of the map nodes are determined by the degree value; a small size with a low degree is shown in blue, and a large size with a high degree is shown in red 
Table 2 Significance levels of the six core genes

\begin{tabular}{llllllll}
\hline Probe ID & Gene & logFC & AveExpr & t & P.Value & adj.P.Val & B \\
\hline 1554997_a_at & PTGS2 & -1.24882193 & 7.442496698 & -4.80511919 & $5.16876 \mathrm{E}-06$ & 0.000540758 & 3.864008467 \\
205067_at & IL1B & -0.698039222 & 10.48159159 & -3.076232156 & 0.002673054 & 0.027765348 & -1.836198698 \\
243213_at & STAT3 & -0.577032001 & 6.221396911 & -4.567697671 & $1.34764 \mathrm{E}-05$ & 0.000947071 & 2.97764575 \\
203936_S_at & MMP9 & -0.605072277 & 8.96615712 & -3.402170393 & 0.000946897 & 0.014217238 & -0.90791425 \\
227697_at & SOCS3 & -0.688732269 & 9.644451144 & -3.821735382 & 0.000224833 & 0.005527334 & 0.394351896 \\
204470_at & CXCL1 & -0.665002791 & 7.462440332 & -3.129305647 & 0.002268543 & 0.025006572 & -1.690241743
\end{tabular}

six genes (PTGS2, IL1B, STAT3, MMP9, SOCS3 and CXCL1) and two miRNAs (miR-320b and miR-320d) as worth exploring due to their core position in the network and the functional enrichment.

Some of the above-mentioned DE-miRNAs were confirmed to be involved in the pathophysiological process of stroke, including neurogenesis (miR-30-5p [19]), neuroprotection (miR-223 [20], miR-424 [21] and miR-106$5 p$ [22]) and angiogenesis (miR-130a [23]). To verify the identified DE-miRNAs in depth, we predicted the target genes and enrichment pathways of the top five DEmiRNAs. ultimately, we speculated that miR-320b and miR-320d were more likely to compensate the pathophysiological process of stroke through the neurotrophin signaling pathway.

The enrichment analysis from the GAD database revealed that traditional risk factors play an important role

Table 3 DE-miRNAs

\begin{tabular}{llllll}
\hline miRNA ID & P.Value & $\mathrm{t}$ & $\mathrm{B}$ & logFC & Regulation \\
\hline hsa-miR-140-3p & 0.00194 & 4.12368 & -2.43 & 4.81174 & up \\
hsa-miR-320b & 0.0024 & 3.9932 & -2.51 & 4.83185 & up \\
hsa-miR-320d & 0.00309 & 3.83947 & -2.61 & 4.96841 & up \\
hsa-miR-320e & 0.00438 & 3.63176 & -2.75 & 4.64221 & up \\
hsa-miR-5100 & 0.00654 & -3.39579 & -2.91 & -1.86679 & down \\
hsa-miR-30d-5p & 0.00683 & 3.37023 & -2.93 & 5.55511 & up \\
hsa-miR-320a & 0.00717 & 3.34187 & -2.95 & 4.38005 & up \\
hsa-miR-4454 & 0.00893 & -3.21442 & -3.05 & -2.17604 & down \\
hsa-miR-3195 & 0.00959 & 3.17256 & -3.08 & 4.14651 & up \\
hsa-miR-6090 & 0.0112 & 3.08296 & -3.15 & 1.03161 & up \\
hsa-miR-2392 & 0.01162 & 3.06168 & -3.16 & 3.29409 & up \\
hsa-miR-642b-3p & 0.01246 & 3.02149 & -3.19 & 4.24362 & up \\
hsa-miR-106b-5p & 0.01257 & 3.01629 & -3.2 & 4.97951 & up \\
hsa-miR-4687-3p & 0.01307 & 2.99373 & -3.21 & 0.33535 & up \\
hsa-miR-149-3p & 0.01621 & -2.86985 & -3.31 & -0.34278 & down \\
hsa-miR-345-5p & 0.01659 & -2.85646 & -3.32 & -0.34535 & down \\
hsa-miR-3135b & 0.01675 & 2.85072 & -3.33 & 3.88918 & up \\
hsa-miR-622 & 0.01766 & -2.82032 & -3.35 & -0.35244 & down \\
hsa-miR-513c-5p & 0.01849 & -2.79399 & -3.37 & -0.35776 & down \\
hsa-miR-583 & 0.01901 & -2.7779 & -3.38 & -0.36108 & down \\
\hline
\end{tabular}

in the onset of stroke, including type 2 diabetes, heart disease, atherosclerosis, high HDL-C levels and Obesity. In addition, the KEGG enrichment and GSEA revealed that DE genes were primarily involved in the TNF and SLE pathways as well as in atypical microbial infection (virus, amoebiasis, legionellosis, vibrio cholerae); these findings were consistent with the inflammatory and immune dysfunction categories in the results of the GO enrichment with biological process. Inflammation and immunity are key elements of the pathobiology of stroke. The immune system gets involved in the cerebral ischemic damage, and the damaged brain in turn suppresses immunity, thereby increasing the incidence of infections and poor outcomes. Inflammation signaling participates in the overall process of the ischemic cascade, from the initial damaging events triggered by arterial occlusion to the late regenerative process underlying post-ischemic tissue repair [24]. Combining the results of the two PPI networks, five core genes (PTGS2, IL1B, SOCS3, MMP9 and $C X C L 1$ ) were linked to inflammation and immunity.

In the early stage of stroke, damaged neurons and endothelial cells produce COX-2 (encoded by PGTS2), which is an important source of prostaglandin. Prostaglandin is a vital inflammatory mediator that launches inflammation and alters the permeability of the blood brain barriers [25]. Subsequently, the microglia in the central nervous system and macrophages in the perivascular space release cytokines, such as TNF and IL-1 $\beta$ (encoded by $I L-1 B$, providing further signals to guide leukocyte migration across the vascular wall) [26]. The chemokine ( $\mathrm{C}-\mathrm{X}-\mathrm{C}$ motif) ligand $1(C X C L 1)$ is a small cytokine belonging to the CXC chemokine family and is expressed in epithelial cells, macrophages and neutrophils, helping to recruit leukocytes to the damaged endothelial cells [27]. When leukocytes migrate from the open blood brain barriers to the vessel extracellular matrix, Matrix metallopeptidase $9(M M P-9)$ is activated to break down the extracellular matrix and remodel it to facilitate the migration of leukocyte to the focus. In the period following stroke, the inflammation responses that clear the dead cells also cause tissue damage and the activation of innate and adaptive immunity [28]. Moreover, recent research shows that vascular endothelial growth factor (VEGF) is crucial for post-ischemic angiogenesis 


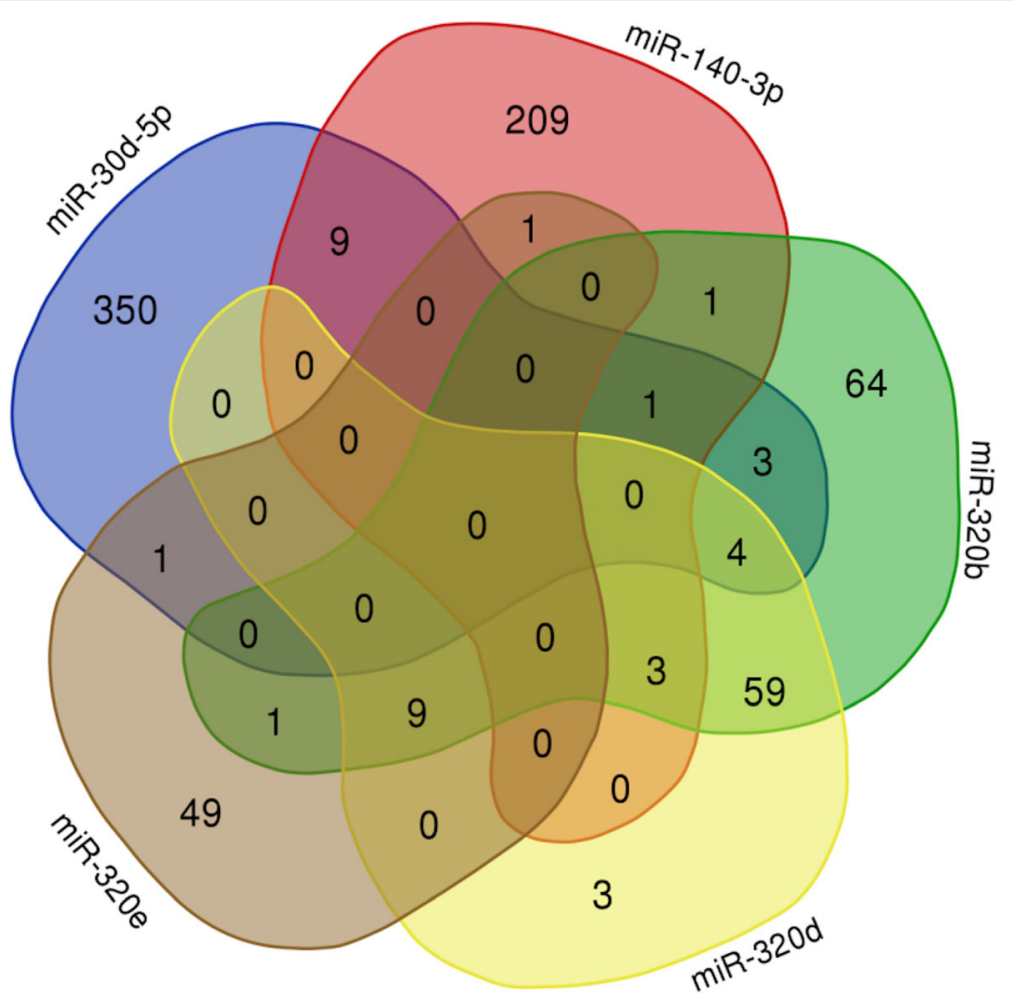

Fig. 8 Intersection of the target genes of the top five miRNAs

and is produced by activated astrocytes; fully functional VEGF may require MMPs, suggesting a link between inflammatory cells and angiogenesis [29]. As for signal transducer and activator of transcription 3 (STAT3), this neuroprotective factor is essential for the differentiation of Th17 cells and for maintaining the ability to generate antibodies of adaptive immunity.

However, high expression of STAT3 in microglia was shown to play a critical role in mediating Hcy-induced microglia activation and neuroinflammation in a rat middle cerebral artery occlusion (MCAO) model [30]. Therefore, the role of $S A T A 3$ in stroke is still controversial. In addition, suppressor of cytokine signaling 3 (SOCS3) has been identified to have an emerging role linking central insulin resistance and Alzheimer's disease, but the relationship between SOCS3 and stroke has not been studied sufficiency [31].

The KEGG and GAD enrichment analyses for DEgenes revealed that the DE-genes related to the following three types of diseases: (1) type 2 diabetes, atherosclerosis and coronary artery disease, which all represent vascular endothelial injury caused by metabolic disorders and fatty acid accumulation; all are considered high risk factors for stroke; (2) SLE and asthma, which both involve excessive inflammation and immune response; and (3) microbial infections (helicobacter pylori, virus, amoebiasis, legionellosis, vibrio cholerae), which are all a direct consequence of immunosuppression in late post- ischemic stroke. The prevailing conclusion is that stroke is a polygenic condition made our integrated analysis more effective and valid.

Here, we proposed that the SLE pathway may be a rare stroke-related pathway. This pathway has been reportedly linked to cerebral lupus, especially epilepsy and acute psychotic disorder [32]. It has been reported that stroke represents one of the most severe complication, with an occurrence rate between 3 to $20 \%$, particularly in the first 5 years of diseases [33]. The mechanisms underlying SLE and stroke involve the expression of aPL (a common SLE antibody) on endothelial surfaces, which leads to the release of pro-inflammatory cytokines and the upregulation of adhesion molecules [34]. However, it is not clear how these antibodies trigger thrombosis. In our study, we outlined the upregulated proteins of the SLE pathway. In the generation stage of auto-antibodies, the overexpression of $\mathrm{CD} 80 / 86$ in antigen-presenting cells accelerates the transduction from Th0 cells to Th2 cells. Then, Th2 cells assist the B cells in producing more antibodies. In the effective stage, on the one hand, $\mathrm{C} 4 / \mathrm{C} 1 \mathrm{q} / \mathrm{C} 5$ in the complement pathway is activated by the antigen-antibody complex to form the MAC (membrane attack complex), leading to vascular endothelial injury. On the other hand, recruited neutrophil granulocytes and macrophages secrete cathepsin, leading to tissue damage in the brain [35]. 


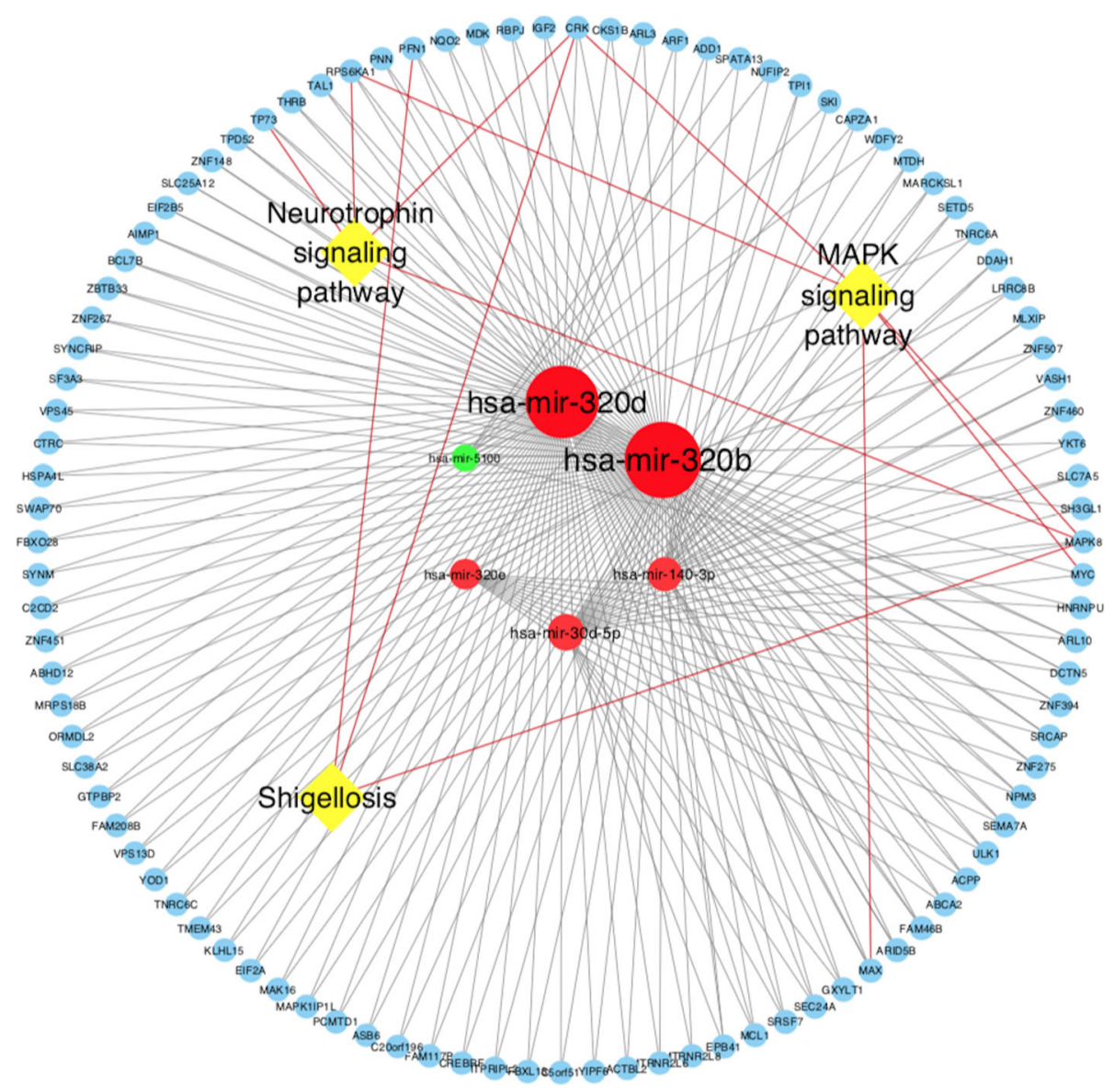

Fig. 9 Outline of the interactions among the significant KEGG pathways, DE genes and miRNAs

Another issue we would like to explore is the atypical infections found in our study. Infection occurrence is an critical trigger that precedes up to one third of ischemic strokes, and infections that present subsequent to ischemic stroke also complicate one third of the cases and bring about worse outcomes [36]. One of the largest studies, which included 19,063 first-time stroke patients, indicated that the risk of stroke was highest during the first 3 days after the diagnosis of respiratory tract infection ( $\mathrm{IR}=3.1995 \% \mathrm{CI} 2.81-3.62)$ or urinary tract infection ( $\mathrm{IR}=2.7295 \% \mathrm{CI} 2.32-3.20$ ). However, in the following PASS (preventive antibiotics in stroke study), preventive ceftriaxone did not improve functional outcomes in patients with acute stroke [37]. In our study, the most significant infections were Helicobacter pylor$i(H P)$, virus and certain atypical microorganisms, including amoebiasis, legionellosis and vibrio cholerase, which were not covered by ceftriaxone. Several retrospective analyses have shown that HP infection is associated with stroke, but their conclusions were contradictory [38]. In addition, several viral infections (cytomegalovirus, Herpes simplex virus 1, varicella zoster virus, hepatitis C virus and human immunodeficiency virus) have been implicated in increasing the risk of ischemic stroke. However, the more atypical infections found in our study were not covered by ceftriaxone, which may account for the negative results in PASS. Notably, there is a lack of research on the relationships between these infections and stroke. With the rise in research on the gutbrain axis, it has been shown that stroke promotes the translocation and dissemination of selective bacterial strains that originate from the host intestinal microbiota [39]. Moreover, the velocity of stroke induces intestinal barrier dysfunction and permeability more rapidly than the dissemination of orally ingested bacteria to peripheral tissues. These studies raised our awareness that we should pay more attention to the relationship between stroke and inapparent infections of the digestive system [40].

Our study has several limitations. The first was the sample types of mRNA datasets. In the original protocol, we aimed to obtain data from blood, cerebrospinal fluid and brain samples in order to restore the differences in gene expression from the periphery and center to pathology. However, due to the limitation of datasets and the inaccessibility of the raw data, the sample type was 
restricted to blood sample data only. The second was the lack of compare of two miRNA datasets from different platform. We just listed the results of the individual analysis together but could not overcome the differences between the platforms to do the fusion analysis. Jung $\mathrm{KC}$ and Daniel R $[41,42]$ developed a random effects model shown to be appropriate for gene express datasets, independent of the method and technology used(ie, spotted cDNA versus oligonucleotide). What's more, By using this method, dataset from different experiment type and platform could cross-verify each other, and that will greatly increased the credibility of microarray analysis. And we were looking forward to see more breakthrough in miRNA analysis in the future. The last shortcoming is that the causal relationship between the novel biomarkers and stoke can only be predicted by theoretical analysis rather than through prospective study. Therefore, we will keep monitoring the progress in stroke research. Further investigations are warranted to confirm whether our novel biomarkers are potential prognostic predictors or therapeutic targets in stroke.

\section{Conclusion}

Our integrated analysis of stroke genomics provides abundant resources for further explorations of the role of target genes and miRNA in ischemic stroke. Six significantly upregulated genes (PTGS2, IL1B, STAT3, $M M P 9$, SOCS3 and CXCL1) and two significantly upregulated miRNAs (miR-320b and miR-320d) were identified as potentially useful clinical diagnostic markers. Systemic Lupus Erythematosus pathways and atypical pulmonary and digestive infections may participate the pathogenesis of stroke; therefore, these topics warrant further study.

\section{Supplementary information}

Supplementary information accompanies this paper at https://doi.org/10. 1186/s12881-020-00994-3.

Additional file 1: Table S1. The DE-miRNA of two groups of miRNA dataset.

Additional file 2: Fig. S1. The boxplot of miRNA sample.

\section{Acknowledgements}

I wish to express my appreciation for comments and suggestions by reviewers Mr. Andrej Kastrin and Mr. Seyit Ali Kayis. Copyright and modify permission of KEGG pathway imagine (Fig. 6 (map05322 Systemic lupus erythematosus on KEGG)) is granted to BMC Medical Genetics to publish from Miwako Matsumoto Kanehisa Laboratories.

\section{Authors' contributions}

QX and LY designed the protocol. XZ, SP, and JS collected the data. SP and $X C$ standardized the raw data. QX and YD analyzed the experimental results. QX and LY wrote the manuscript. The author(s) read and approved the final manuscript.

\section{Funding}

This project was supported by grants from the SanMing Project of National Clinical Research Center for Geriatric Diseases Shenzhen Center in Peking University Shenzhen Hospital No. SZSM201812096) and Peking University Shenzhen Hospital Core Research Funding (JCYJ2018012).

\section{Availability of data and materials}

The datasets generated and/or analyzed during the current study are available from the GEO database and can be download through the following link.

https://www.ncbi.nlm.nih.gov/geo/query/acc.cgi?acc=GSE66724 https://www.ncbi.nlm.nih.gov/geo/query/acc.cgi?acc=GSE22255 https://www.ncbi.nlm.nih.gov/geo/query/acc.cgi?acc=GSE86291 https://www.ncbi.nlm.nih.gov/geo/query/acc.cgi?acc=GSE58294 https://www.ncbi.nlm.nih.gov/geo/query/acc.cgi?acc=GSE55937

\section{Ethics approval and consent to participate}

No applicable. (The study was carried out by collecting information freely available in the public database (GEO database) and the analysis of datasets, either open source or obtained from other researchers where the data are properly anonymised and informed consent was the time of original data collection.)

\section{Consent for publication}

Not applicable.

\section{Competing interests}

The authors declare that they have no competing interests. No competing financial interests exist.

\section{Author details}

'Department of Neurology, Peking University Shenzhen Hospital, Shenzhen, China. ${ }^{2}$ Department of Clinical Medicine, Shantou University Medical College, Shantou, China. ${ }^{3}$ National Clinical Research Center for Geriatric Diseases Shenzhen Center, Peking University Shenzhen Hospital, Shenzhen, China.

Received: 6 October 2019 Accepted: 9 March 2020

Published online: 30 March 2020

\section{References}

1. Global, regional, and national life expectancy, all-cause mortality, and causespecific mortality for 249 causes of death, 1980-2015: a systematic analysis for the Global Burden of Disease Study 2015. Lancet. 2016;388(10053):1459-544.

2. Feigin $\mathrm{VL}$, Roth $\mathrm{GA}$, Naghavi $M$, et al. Global burden of stroke and risk factors in 188 countries, during 1990-2013: a systematic analysis for the global burden of disease study 2013. Lancet Neurol. 2016;15(9):913-24.

3. Benjamin EJ, Virani SS, Callaway CW, et al. Heart disease and stroke Statistics-2018 update: a report from the American Heart Association. Circulation. 2018;137(12):e67-e492.

4. Lindgren A, Lovkvist $\mathrm{H}$, Hallstrom B, et al. Prevalence of stroke and vascular risk factors among first-degree relatives of stroke patients and control subjects. A prospective consecutive study. Cerebrovasc Dis. 2005;20(5):381-7.

5. Seshadri S, Beiser A, Pikula A, et al. Parental occurrence of stroke and risk of stroke in their children: the Framingham study. Circulation. 2010;121(11): 1304-12.

6. Møller A, Rasmussen L, Ledet T. Plasma lipoprotein composition in type 2 diabetic patients. Scand J Clin Lab Invest. 1987;47(7):731-8. 
7. Schweizer S, Meisel A, Märschenz S. Epigenetic mechanisms in cerebral ischemia. J Cereb Blood Flow Metab. 2013;33(9):1335-46.

8. Schadt EE, Lamb J, Yang $X$, et al. An integrative genomics approach to infer causal associations between gene expression and disease. Nat Genet. 2005; 37(7):710-7.

9. Owolabi M, Peprah $\mathrm{E}_{1} \mathrm{Xu} \mathrm{H}$, et al. Advancing stroke genomic research in the age of trans-Omics big data science: emerging priorities and opportunities. J Neurol Sci. 2017;382:18-28.

10. Johnson WE, Li C, Rabinovic A. Adjusting batch effects in microarray expression data using empirical Bayes methods. Biostatistics. 2007;8(1): $118-27$.

11. Tseng GC, Ghosh D, Feingold E. Comprehensive literature review and statistical considerations for microarray meta-analysis. Nucleic Acids Res. 2012;40(9):3785-99.

12. Huang DW, Sherman BT, Tan Q, et al. DAVID Bioinformatics Resources: expanded annotation database and novel algorithms to better extract biology from large gene lists. Nucleic Acids Res. 2007;35(Web Server issue): W169-75.

13. Huang DW, Sherman BT, Lempicki RA. Bioinformatics enrichment tools: paths toward the comprehensive functional analysis of large gene lists. Nucleic Acids Res. 2009;37(1):1-13.

14. Kanehisa M, Goto S. KEGG: Kyoto encyclopedia of genes and genomes. Nucleic Acids Res. 2000;28:27-30

15. Szklarczyk D, Franceschini A, Wyder S, et al. STRING v10: protein-protein interaction networks, integrated over the tree of life. Nucleic Acids Res. 2015:43(Database issue):D447-52.

16. Shannon P, Markiel A, Ozier O, et al. Cytoscape: a software environment for integrated models of biomolecular interaction networks. Genome Res. 2003; 13(11):2498-504

17. Subramanian A, Tamayo P, Mootha VK, et al. Gene set enrichment analysis: a knowledge-based approach for interpreting genome-wide expression profiles. Proc Natl Acad Sci U S A. 2005;102(43):15545-50.

18. Wang $X$, Kang DD, Shen $K$, et al. An R package suite for microarray metaanalysis in quality control, differentially expressed gene analysis and pathway enrichment detection. Bioinformatics. 2012;28(19):2534-6.

19. Mellios N, Huang HS, Grigorenko A, Rogaev E, Akbarian S. A set of differentially expressed miRNAs, including miR-30a-5p, act as posttranscriptional inhibitors of BDNF in prefrontal cortex. Hum Mol Genet. 2008;17(19):3030-42.

20. Harraz MM, Eacker SM, Wang X, Dawson TM, Dawson VL. MicroRNA-223 is neuroprotective by targeting glutamate receptors. Proc Natl Acad Sci U S A. 2012;109(46):18962-7.

21. Liu P, Zhao H, Wang R, et al. MicroRNA-424 protects against focal cerebral ischemia and reperfusion injury in mice by suppressing oxidative stress. Stroke. 2015:46(2):513-9.

22. Li P, Shen M, Gao F, et al. An Antagomir to MicroRNA-106b-5p ameliorates cerebral ischemia and reperfusion injury in rats via inhibiting apoptosis and oxidative stress. Mol Neurobiol. 2017;54(4):2901-21.

23. Chen Y, Gorski DH. Regulation of angiogenesis through a microRNA (miR130a) that down-regulates antiangiogenic homeobox genes GAX and HOXA5. Blood. 2008;111(3):1217-26.s.

24. ladecola C, Anrather J. The immunology of stroke: from mechanisms to translation. Nat Med. 2011:17(7):796-808.

25. Lemaitre RN, Rice K, Marciante K, et al. Variation in eicosanoid genes, non-fatal myocardial infarction and ischemic stroke. Atherosclerosis. 2009;204(2):e58-63.

26. Pan W, Kastin AJ. Tumor necrosis factor and stroke: role of the blood-brain barrier. Prog Neurobiol. 2007:83(6):363-74.

27. Losy J, Zaremba J, Skrobański P. CXCL1 (GRO-alpha) chemokine in acute ischaemic stroke patients. Folia Neuropathol. 2005;43(2):97-102.

28. Ramos-Fernandez M, Bellolio MF, Stead LG. Matrix metalloproteinase-9 as a marker for acute ischemic stroke: a systematic review. J Stroke Cerebrovasc Dis. 2011;20(1):47-54.

29. Hayakawa K, Nakano T, Irie K, et al. Inhibition of reactive astrocytes with fluorocitrate retards neurovascular remodeling and recovery after focal cerebral ischemia in mice. J Cereb Blood Flow Metab. 2010;30(4):871-82.

30. Chen S, Dong Z, Cheng M, et al. Homocysteine exaggerates microglia activation and neuroinflammation through microglia localized STAT3 overactivation following ischemic stroke. J Neuroinflammation. 2017;14(1):187.

31. Cao L, Wang Z, Wan W. Suppressor of cytokine signaling 3: emerging role linking central insulin resistance and Alzheimer's disease. Front Neurosci. 2018;12:417
32. Honczarenko K, Budzianowska A, Ostanek L. Neurological syndromes in systemic lupus erythematosus and their association with antiphospholipid syndrome. Neurol Neurochir Pol. 2008;42(6):513-7.

33. Saadatnia M, Sayed-Bonakdar Z, Mohammad-Sharifi G, Sarrami AH. The necessity of stroke prevention in patients with systemic lupus erythematosus. J Res Med Sci. 2012;17(9):894-5.

34. Meroni PL, Tincani A, Sepp N, et al. Endothelium and the brain in CNS lupus. Lupus. 2003;12(12):919-28.

35. Rahman A, Isenberg DA. Systemic lupus erythematosus. N Engl J Med. 2008; 358(9):929-39.

36. Emsley HC, Hopkins SJ. Acute ischaemic stroke and infection: recent and emerging concepts. Lancet Neurol. 2008;7(4):341-53.

37. Westendorp WF, Zock E, Vermeij JD, et al. Preventive antibiotics in stroke study (PASS): a cost-effectiveness study. Neurology. 2018;90(18):e1553-60.

38. Chen Y, Segers S, Blaser MJ. Association between helicobacter pylori and mortality in the NHANES III study. Gut. 2013;62(9):1262-9.

39. Stanley D, Mason LJ, Mackin KE, et al. Translocation and dissemination of commensal bacteria in post-stroke infection. Nat Med. 2016;22(11):1277-84.

40. Yang $X$, Gao Y, Zhao X, Tang Y, Su Y. Chronic helicobacter pylori infection and ischemic stroke subtypes. Neurol Res. 2011;33(5):467-72.

41. Rhodes DR, Barrette TR, Rubin MA, et al. Meta-analysis of microarrays: interstudy validation of gene expression profiles reveals pathway dysregulation in prostate cancer[J]. Cancer Res. 2002;62(15):4427-33.

42. Choi JK, Yu U, Kim S, et al. Combining multiple microarray studies and modeling interstudy variation[J]. Bioinformatics. 2003;19(suppl_1):i84-90.

\section{Publisher's Note}

Springer Nature remains neutral with regard to jurisdictional claims in published maps and institutional affiliations.
Ready to submit your research? Choose BMC and benefit from:

- fast, convenient online submission

- thorough peer review by experienced researchers in your field

- rapid publication on acceptance

- support for research data, including large and complex data types

- gold Open Access which fosters wider collaboration and increased citations

- maximum visibility for your research: over $100 \mathrm{M}$ website views per year

At $\mathrm{BMC}$, research is always in progress.

Learn more biomedcentral.com/submissions 